\title{
Prospecção científica e tecnológica de Caryocar coriaceum Wittm.
}

\author{
Scientific and technological prospection of Caryocar coriaceum Wittm. \\ Prospección cientifica y tecnológica de Caryocar coriaceum Wittm.
}

\author{
Vanessa Fernanda da Silva Sousa \\ ORCID: https://orcid.org/0000-0002-8591-5896 \\ Universidade Federal do Piaú, Brasil \\ E-mail: vanessa_fernandaSS@hotmail.com \\ Tony César de Sousa Oliveira \\ ORCID: https://orcid.org/0000-0001-6209-3829 \\ Universidade de São Paulo, Brasil \\ E-mail: tonycsoliveira@usp.br \\ Ivanilza Moreira de Andrade \\ ORCID: https://orcid.org/0000-0001-6059-8540 \\ Universidade Federal do Delta do Parnaíba, Brasil \\ E-mail: ivanilzaandrade@hotmail.com
}

\begin{abstract}
Resumo
Caryocar coriaceum Wittm. é uma espécie frutífera, nativa e endêmica do cerrado brasileiro, amplamente utilizada no ramo alimentício e medicinal. Nessa pesquisa, objetivou-se realizar uma prospecção científica e tecnológica sobre $C$. coriaceum a partir da análise de patentes e artigos científicos indexados em bases de dados nacionais (INPI) e internacionais (WEB OF SCIENCE, EPO e DII). Foram registrados 29 artigos científicos na Web of Science, sendo 2011, 2015 e 2020 os anos com maior número de publicações $(n=4$, cada). Na prospecção tecnológica, o número de patentes depositadas com a espécie é considerado baixo $(\mathrm{n}=7)$, se comparado à outra espécie do gênero, como Caryocar brasiliense Cambess $(\mathrm{n}=57)$. A EPO foi a base de dados com maior registro de patentes $(\mathrm{n}=4)$, com destaque para invenções com finalidades médicas e cosmetológicas e o Brasil, o principal depositário. Os resultados dessa pesquisa sugerem que, apesar das potencialidades da espécie e do relativo interesse brasileiro, o desenvolvimento científico e tecnológico da espécie encontra-se em lenta ascensão, evidenciando a necessidade de maior incentivo às produções e inovações tecnológicas com ela.
\end{abstract}

Palavras-chave: Pequi; Produção científica; Patente.

\begin{abstract}
Caryocar coriaceum Wittm., a fruit species, is native and endemic to the Brazilian cerrado, widely used both in the food and medicine industry. This study was aimed carried out a scientific and technological forecasting of $C$. coriaceum using analysis of both scientific papers and patents indexed in national (INPI) and international (WEB OF SCIENCE, EPO and DII) databases. In total, 29 papers were registered and analyzed in the Web of Science, being 2011, 2015 and 2020 the years with the highest number of publications $(n=4$, each). With regard to technological prospecting, the number of filed patents found was considered low $(n=7)$, when compared to other species of the same genus, for example, Caryocar brasiliense Cambess $(n=57)$. The EPO was the database with the largest patent registration $(n=4)$. In it, Brazil is the main depositor country with emphasis on medical and cosmetological purposes. These results suggest that, currently, the interest in the species' potential is strictly Brazilian and that the scientific and technological development of the species is still growing slowly, which highlights a need for a greater incentive to production and technological innovations with this species.
\end{abstract}

Keywords: Pequi; Scientific production; Patent.

\section{Resumen}

Caryocar coriaceum Wittm. es una especie frutal, nativa y endémica del cerrado brasileño, ampliamente utilizada en los campos alimentario y medicinal. En esta investigación, el objetivo fue realizar una prospeccnion científica y tecnológica de Caryocar coriaceum basado en el análisis de patentes y artículos científicos indexados en bases de datos nacionales (INPI) e internacionales (WEB OF SCIENCE, EPO y DII). Veintinueve artículos científicos se registraron en la Web of Science, siendo 2011, 2015 y 2020 los años con mayor número de publicaciones $(\mathrm{n}=4$, cada uno). En prospección tecnológica, el número de patentes presentadas sobre la especie se considera bajo $(\mathrm{n}=7)$, en comparación con otra especie del género, como Caryocar brasiliense $(n=57)$. La EPO fue la base de datos con mayor registro de patentes $(n=4)$, con énfasis en invenciones con fines médicos y cosmetológicos, y Brasil, el principal depositario. Los resultados de esta investigación sugieren que, a pesar del potencial de la especie y del 
relativo interés brasileño, el desarrollo científico y tecnológico de la especie está en lento ascenso, destacando la necesidad de un mayor incentivo a la producción e innovaciones tecnológicas con ella.

Palabras clave: Pequi; Producción científica; Patentar.

\section{Introdução}

No ranking dos dezessete países que abrigam $70 \%$ da riqueza de espécies do planeta, o Brasil se destaca por ser o mais megadiverso, englobando de 15 a $25 \%$ de todas as espécies vegetais e por apresentar elevada taxa de endemismo e dispersão em biomas característicos (Joly, 2011; Scarano \& Ceotto, 2016).

É importante destacar que, os elevados índices de diversidade biológica das regiões neotropicais estão relacionados a singularidades ocorridas durante o processo de formação geológica da região, fato que explica a megadiversidade de espécies encontradas em países como Brasil, Colômbia, Peru e Equador (Räsänen, et al., 1995) e que reforça a importância e elevado potencial dos recursos biológicos nesses países.

Dentre os elementos com elevado potencial biológico, a flora se apresenta como importante fonte de substâncias biologicamente ativas, com amplas possibilidades no desenvolvimento de produtos cosméticos, agroquímicos e suplementos alimentares, constituem ainda, a principal matéria-prima na produção de fitoterápicos e outros medicamentos (Sandes \& Diblasi, 2000; Brasil, 2006; Joly, 2011). As espécies vegetais são conhecidas pela humanidade há centenas de anos e utilizadas na medicina tradicional, como remédios caseiros e comunitários no tratamento de diversas enfermidades (Brasil, 2006; Oliveira, et al., 2006).

Dentre diversas espécies de interesse, características dos biomas brasileiros, podemos destacar Caryocar coriaceum Wittm, conhecida popularmente como Pequi, um táxon nativo e endêmico do Cerrado da região nordeste do país (Prance \& Silva, 1973; Sousa-Junior, 2018; Prance \& Pirani, 2020). O fruto do pequi pode ser aproveitado integralmente, no entanto, a polpa constitui seu principal produto, composto de mesocarpo interno rico em carboidratos $(57,83 \%)$, lipídeos (33,53\%), fibras totais $(5,29 \%)$ e proteínas (3,28\%) (Ramos \& Souza, 2011; Brasil, 2019).

Devido à disponibilidade desse recurso vegetal e à versatilidade de usos, a espécie C. coriaceum apresenta grande importância socioambiental para as comunidades locais, onde é comumente utilizada na atividade econômica e alimentícia da população, tanto na forma in situ como na comercialização de pratos regionais (Oliveira, et al., 2008; Pianovski, et al., 2008; Souza, et al., 2013). Além de fins culinários, a espécie é amplamente utilizada, de maneira extrativista, na medicina popular, indústria farmacêutica, cosmética, de combustíveis, além das aplicações artesanais (Oliveira, et al. 2008; Lorenzi, 2014). Vale ressaltar ainda que a sua exploração alimentar e econômica é restrita a alguns meses do ano devido ao seu caráter sazonal (Sousa, et al., 2014).

Apesar do elevado potencial econômico da espécie, seu caráter endêmico, dificuldade de propagação decorrente de peculiaridades morfo-anatômicas, e destruição acelerada dos ecossistemas do seu bioma de ocorrência (Machado, et al., 2004; Prance \& Silva, 2007), ainda são poucas as publicações científicas sobre a espécie, se comparadas a outras do gênero, como Caryocar brasiliense Cambess., e no caso de prospecção científica, os estudos são inexistentes, demonstrando a necessidade e urgência na realização de estudos que possam prover mais informações sobre ela.

Nessa perspectiva, a realização de prospecção científica e tecnológica pode ser considerada uma estratégia sumária na ampliação do conhecimento científico acerca de C. coriaceum, uma vez que, por meio dessa metodologia é possível traçar o perfil das pesquisas que estão sendo realizadas com a espécie até o momento, podendo a análise de seus resultados, revelar os aspectos que têm sido mais explorados ao longo do tempo, permitindo projetar possíveis lacunas científicas existentes. Além disso, segundo Kupfer \& Tigre (2004), as informações presentes nos pedidos de patentes servem como bons indicadores de atividades relacionadas a Pesquisa, Desenvolvimento e Inovação e sobre avanços tecnológicos na área da ciência e tecnologia. 
Diante do contexto apresentado, o objetivo do estudo foi realizar a prospecção científica e tecnológica de $C$. coriaceum, a partir da análise documental de patentes e artigos científicos em bases de dados, a fim de que se possa traçar o atual panorama de publicações e inovações tecnológicas relacionadas à espécie.

\section{Metodologia}

Conforme define Kupfer \& Tigre (2004), a prospecção tecnológica envolve esforços sistemáticos no mapeamento do desenvolvimento científico e tecnológico futuro, capaz de influenciar significativamente uma indústria, economia e sociedade e que, segundo Tigre (2006), ainda permite analisar atores e fatores imbuídos no processo de inovação, suas interrelações, a fim de que se possa predizer, caracterizar e compreender potencialidades e efeitos das mudanças tecnológicas.

Partindo dessa perspectiva, fez-se uma análise e quantificação das publicações científicas e inovações tecnológicas a partir da consulta às bases de dados Web of Science, Instituto Nacional de Propriedade Intelectual (INPI), European Patente Office (Espacenet) e Derwent Innovations Index ${ }^{\circledR}$ (DII), a partir da inserção do descritor genérico "Pequi”, com ênfase em produções do tipo "article". Fez-se o refinamento inserindo a palavra "Caryocar" e posteriormente o termo "Caryocar coriaceum". Por fim, fez-se uma busca com o descritor "Caryocar brasiliense" a fim de se estabelecer um comparativo de produções entre as duas espécies.

Os artigos encontrados na Web of Science foram analisados conforme número e ano das publicações, idioma, país de origem, número de artigos por periódico, número de artigos por autoria, instituições vinculadas às pesquisas, áreas da pesquisa, número de citações dos artigos por ano e por autoria. Já na análise dos pedidos de patentes envolvendo Caryocar coriaceum, analisou-se a aplicação tecnológica da espécie, o perfil do depositante, país de origem da patente, ano de depósito e Classificação Internacional de Patentes (CIP). Ambas as buscas ocorreram em março de 2021, sem recorte temporal. Os dados encontrados foram tratados e compilados no Microsoft Excel 2019.

\section{Resultados e Discussão}

\subsection{Prospecção científica}

$\mathrm{Na}$ prospecção científica realizada na base de dados Web of Science, pode-se observar maior número de publicações utilizando o descritor "pequi", com total de 289 artigos. Com o refinamento para o termo "Caryocar" obteve-se 236 artigos publicados. Refinando com o descritor "Caryocar brasiliense" a ocorrência foi de 195 artigos. Já para descritor "Caryocar coriaceum", que direciona aos estudos com espécie-alvo da pesquisa, esse número é reduzido para aproximadamente 15\%, em relação ao número de pesquisas realizadas com a espécie C. brasiliense (Tabela 1).

Tabela 1: Número de artigos por descritores.

\begin{tabular}{ccc}
\hline Base de dados & Descritor & $\mathbf{N}^{\mathbf{0}}$ de artigos \\
\hline \multirow{2}{*}{ Web of Science } & Pequi & 289 \\
\cline { 2 - 3 } & Caryocar & 236 \\
\cline { 2 - 3 } Total & Caryocar brasiliense & 195 \\
\hline & Caryocar coriaceum & 29 \\
\hline
\end{tabular}

Fonte: Autores (2021). 
A pouca expressividade de estudos com a espécie $C$. coriaceum já era esperado, tendo em vista que se trata de uma espécie endêmica, com distribuição geográfica limitada no Brasil, fatores que podem estar contribuindo para o pouco conhecimento e desenvolvimento científico e tecnológico da espécie. Por outro lado, esse dado aponta para a necessidade e urgência no avanço científico com o fruto, já que segundo Myers, et al. (2000) e Williams, et al. (2011) a espécie tem ocorrência em um dos 35 hotspots mundiais de biodiversidade, que são áreas prioritárias para conservação devido ao alto índice de diversidade e endemismo de espécies e que paralelamente apresentam elevada proporção de habitats degradados (Mittermeier, et al., 2011; Galindo-Leal, 2005).

Complementando os dados anteriores, verificou-se que do total de artigos sobre o C. coriaceum $(\mathrm{n}=29)$, todos são de origem brasileira, com participação de pesquisadores de outros países, como Chile, Itália, Holanda, Peru e Espanha, sendo $82,8 \%$ escritos em língua inglesa e 17,2\% em língua portuguesa, sugerindo que, embora a espécie apresente distribuição restrita ao cerrado brasileiro e poucos trabalhos tenham sido desenvolvidos até o momento, há um grande possibilidade de alcance a um número maior de leitores, uma vez que o inglês é a língua universal.

Analisando as áreas de concentração das pesquisas, evidenciou-se maior destaque para a Agricultura e Farmacologia, ambas com nove artigos, seguidas de Biologia Vegetal e Química, com $(n=8)$ e $(n=7)$, respectivamente. Em menores proporções, encontrou-se as áreas Medicina Complementar Integrativa ( $n=5)$, Ecologia de Ciências Ambientais $(n=4)$, Tecnologia da Ciência Alimentar $(n=4)$, Conservação da Biodiversidade $(n=2)$, Science Outros tópicos de tecnologia $(n=2)$ e Engenharia $(n=1)$. Ressalta-se que um mesmo trabalho pode ser classificado em mais de uma área de pesquisa, levando em consideração as abordagens exploradas nas publicações. Esse resultado demonstra que, apesar da diversidade de áreas relacionadas nas pesquisas, existe correlação entre todas elas, sendo algumas complementares e ainda que a maioria dos trabalhos tem buscado explorar e/ou identificar potencialidades farmacológicas da planta, corroborando com Calixto (2003), que afirma que os produtos naturais comumente são utilizados como fonte de matéria-prima na produção de moléculas complexas de interesse na área de farmacologia.

A Figura 1 retrata a trajetória de publicações com a espécie na Web of Science. Observou-se que, 2011, 2015 e 2020 foram os anos que se destacaram quanto ao número de publicações, com total de quatro em cada ano e que ao longo do período analisado, 2012 foi o único ano em que a base de dados não registrou trabalhos com a espécie.

Figura 1: Número de publicações por ano.

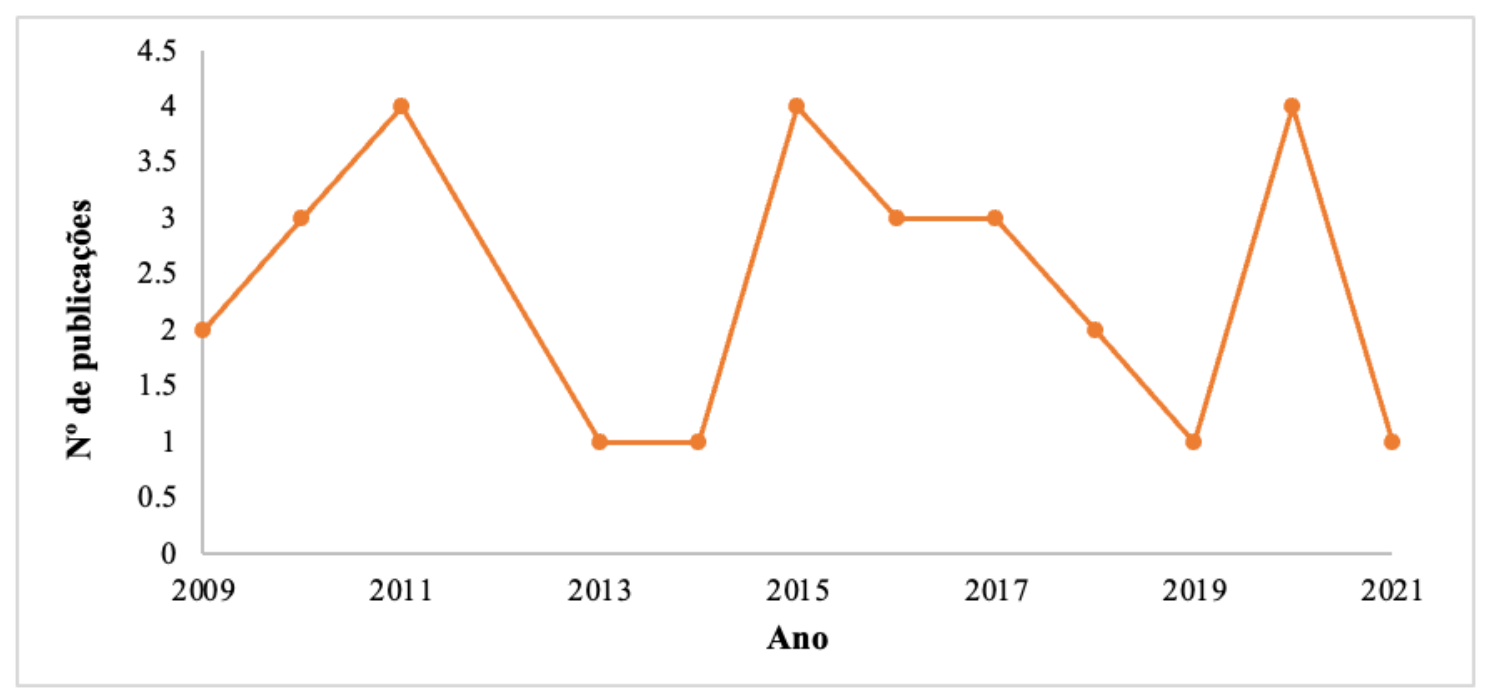

Fonte: Autores (2021). 
Nota-se que, mesmo em anos de pico, o número de publicações não ultrapassa quatro (Figura1), o que nos permite inferir que há uma constância no quadro de publicações e nos intervalos de tempo entre elas. Além disso, é importante destacar que, os estudos com espécie são relativamente recentes, com pouco mais de uma década, apesar de a espécie ter sido incluída na Lista Vermelha com estado de conservação classificado como em perigo (EN) em 1998.

As publicações envolvendo $C$. coriaceum estão distribuídas em vinte e um periódicos, entre os quais tiveram destaque o Journal of Ethnopharmacology ( $n=5)$, Revista Brasileira de fruticultura ( $n=4)$ e Natural Product Research $(n=2)$. Aos demais, as publicações foram de um artigo cada.

Analisando o Qualis dos periódicos com maior número de publicações na Plataforma Sucupira, verificou-se que a Revista Brasileira de fruticultura e a Natural Product Research são classificados como B1 na área de Ciências Ambientais e o Journal of Ethnopharmacology em A1 na mesma área. Esses dois últimos periódicos apresentam escopos semelhantes relacionados a recursos vegetais, sendo o primeiro voltado para publicações no campo da química de produtos naturais e o segundo para investigação experimental das atividades biológicas de substâncias vegetais e animais utilizados na medicina tradicional de culturas passadas e presentes. Já a Revista Brasileira de Fruticultura, como o próprio nome sugere, destina-se à publicação de artigos técnico-científicos e de comunicação científica na área de Fruticultura.

Quanto número de autores envolvidos nas publicações encontrou-se 25 autorias, com destaque para Coutinho $\mathrm{H}$. D. M., Da Costa, J. G. M., Albuquerque U. P. e Meneses I. R. A, que tiveram maior participação em trabalhos, sendo $(n=8),(n=6),(n=4)$ e $(n=4)$, respectivamente (Figura 2). A participação recorrente de autores em trabalhos distintos, evidencia os pesquisadores que mais têm se dedicado a pesquisar sobre a espécie e que em decorrência disso, estão contribuindo para o avanço do conhecimento sobre ela.

Figura 2: Número de artigos por autoria.
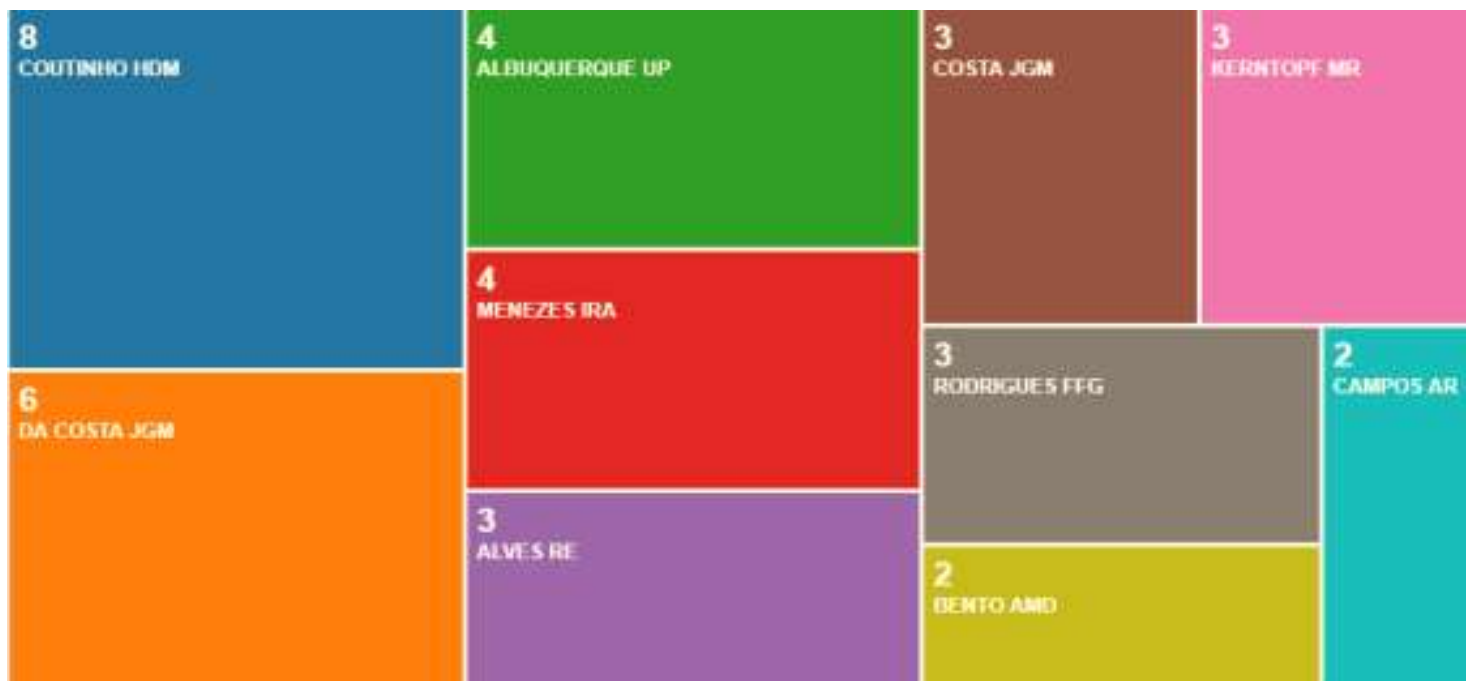

Fonte: Web of Science (2021).

Quanto ao panorama de citações dos artigos em cada ano, notou-se um crescimento ao longo dos anos, tendo 2020, maior destaque, com total de 73 citações (Figura 3). Esse dado revela que, apesar de ainda não haver grande volume de publicações sobre a espécie, o interesse nos artigos relacionados a ela vem crescendo, sobretudo de 2015 para cá. Isso indica que, se a rede de citações seguir o ritmo atual, é possível que em poucos anos as informações e publicações sobre a espécie estejam mais consolidadas e difundidas. É importante frisar que as informações sobre o número de citações 
relativas ao ano de 2021, apresentados aqui, estão incompletas, tendo em vista que a pesquisa foi realizada no primeiro trimestre do presente ano.

Figura 3: Número de citações por ano.

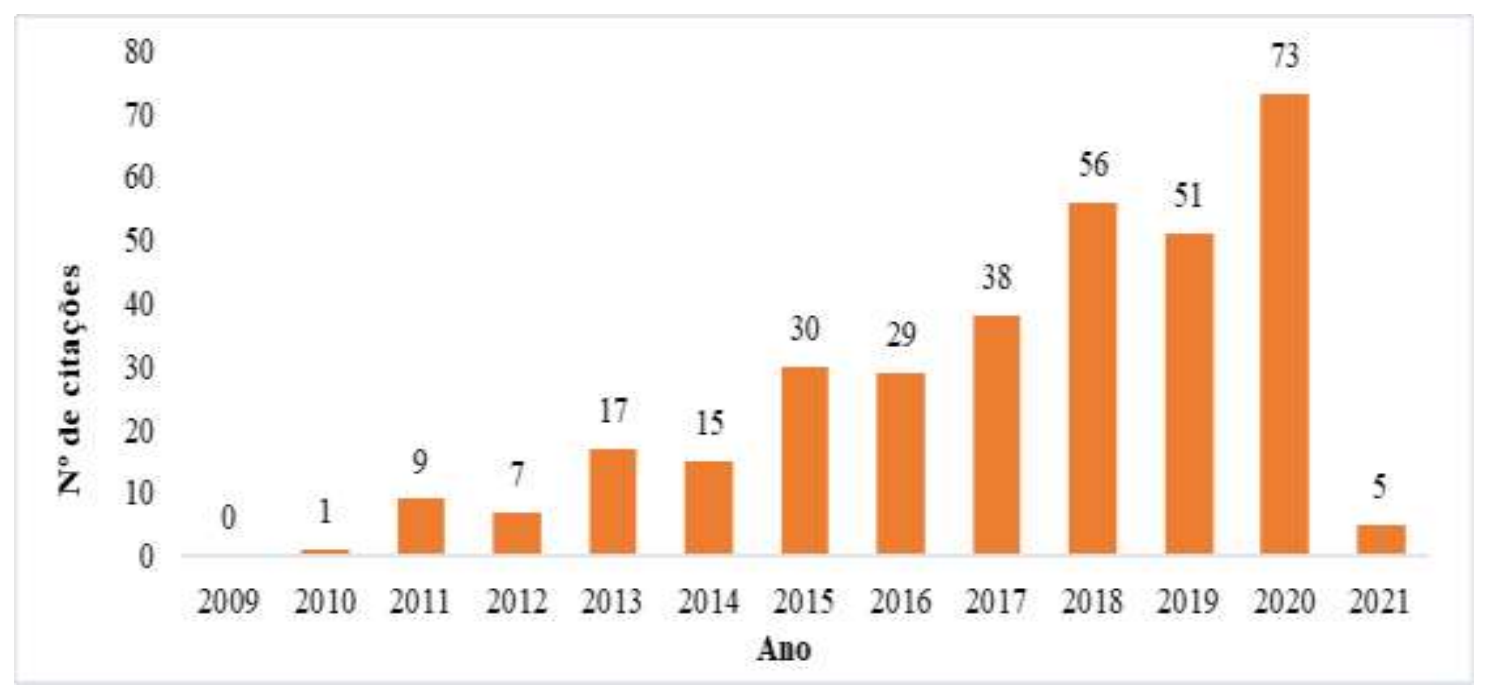

Fonte: Autores (2021).

Complementar a esse dado verificou-se os autores mais citados e que consequentemente contribuíram para o quadro de citações apresentados, foram eles: Saraiva, et al. (2011); Maia de Oliveira, et al. (2010); Sousa Junior, et al. (2013); Pessoa, et al. (2015); Quirino, et al. (2009), que juntos somam 173 citações, o que corresponde à mais de 50\% do total de citações em treze anos.

Os artigos estão vinculados a 25 instituições, sendo as principais, em termos de produção científica, oriundas da região nordeste do Brasil. As três instituições que mais contribuíram para essa disseminação científica foram a Universidade Regional do Cariri $(n=12)$, Universidade Federal do Ceará $(n=8)$ e Universidade Estadual do Ceará $(n=5)$. Esse dado era esperado, tendo em vista que a espécie- alvo desses estudos apresenta ampla distribuição pelos estados do nordeste, fato que pode contribuir para o conhecimento e facilidade de acesso a espécie em questão por parte dos pesquisadores dessa região.

\subsection{Prospecção tecnológica}

A prospecção tecnológica revelou que entre os três bancos de dados analisados, a EPO apresentou maior número de patentes em todos os descritores analisados (Tabela 2). Ao comparar o número de patentes registradas para cada descritor, observa-se que para "Caryocar coriaceum" o número é aproximadamente dez vezes menor que as patentes para o descritor "Caryocar brasiliense", demonstrando que existe considerável disparidade nas inovações tecnológicas para as duas espécies. 
Tabela 2: Número de patentes por descritores em cada base de dados.

\begin{tabular}{ccccc}
\hline DESCRITOR & INPI & EPO & DII & TOTAL \\
\hline Pequi & 25 & 322 & 60 & 407 \\
Caryocar & 8 & 87 & 6 & 101 \\
Caryocar brasiliense & 3 & 51 & 3 & 57 \\
Caryocar coriaceum & 2 & 4 & 1 & 7 \\
\hline TOTAL & 38 & 464 & 70 & 572 \\
\hline
\end{tabular}

Fonte: Autores (2021).

Esse resultado era esperado e se deve à diferença na distribuição geográfica entre as duas espécies, onde $C$. brasiliense apresenta distribuição mais ampla, por todas as regiões brasileiras ao passo em que C. coriaceum tem ocorrência mais restrita aos cerrados do norte, nordeste e centro-oeste do país (CNCFlora, 2011; Amorim, et al., 2012). Para modificar essa estatística, é necessário maior incentivo e investimentos em estudos com a espécie, uma vez que quanto mais estudos sobre ela forem realizados e publicados, mais conhecida será a espécie, o que consequentemente pode gerar maior interesse por ela, reiniciando o ciclo de produção cientifica e tecnológica.

Ao analisar a origem das patentes indexadas, verificou-se que o Brasil se destaca por liderar as patentes envolvendo a espécie $C$. coriaceum nas bases de dados INPI (n=2) e DII (n=1), seguido da Suiça com $(n=2)$ registros na base EPO. Em menor proporção estão os Estados Unidos e Austrália (ambos com n=1) na EPO. De acordo com Rizzini (1978), Pinto (1992) e Silva \& Medeiros-Filho (2006), o Brasil apresenta o maior índice de catalogação de espécies até o presente, sendo, portanto, o país da América latina que mais estuda suas atividades biológicas.

Analisando o histórico de patentes em função do tempo, observou-se o primeiro depósito com a espécie ocorreu em 2006. Além disso, 2007 e 2015 apresentaram-se como os anos mais expressivos em número de patentes indexadas aos bancos de dados INPI e EPO, sendo dois em cada ano (Figura 4). No entanto, é possível perceber que o intervalo entre os depósitos apresenta significativa variação, sobretudo entre esses dois anos, onde o período entre uma patente e outra foi de oito anos.

Figura 4. Patentes registradas com C. coriaceum por ano nas bases de dados.

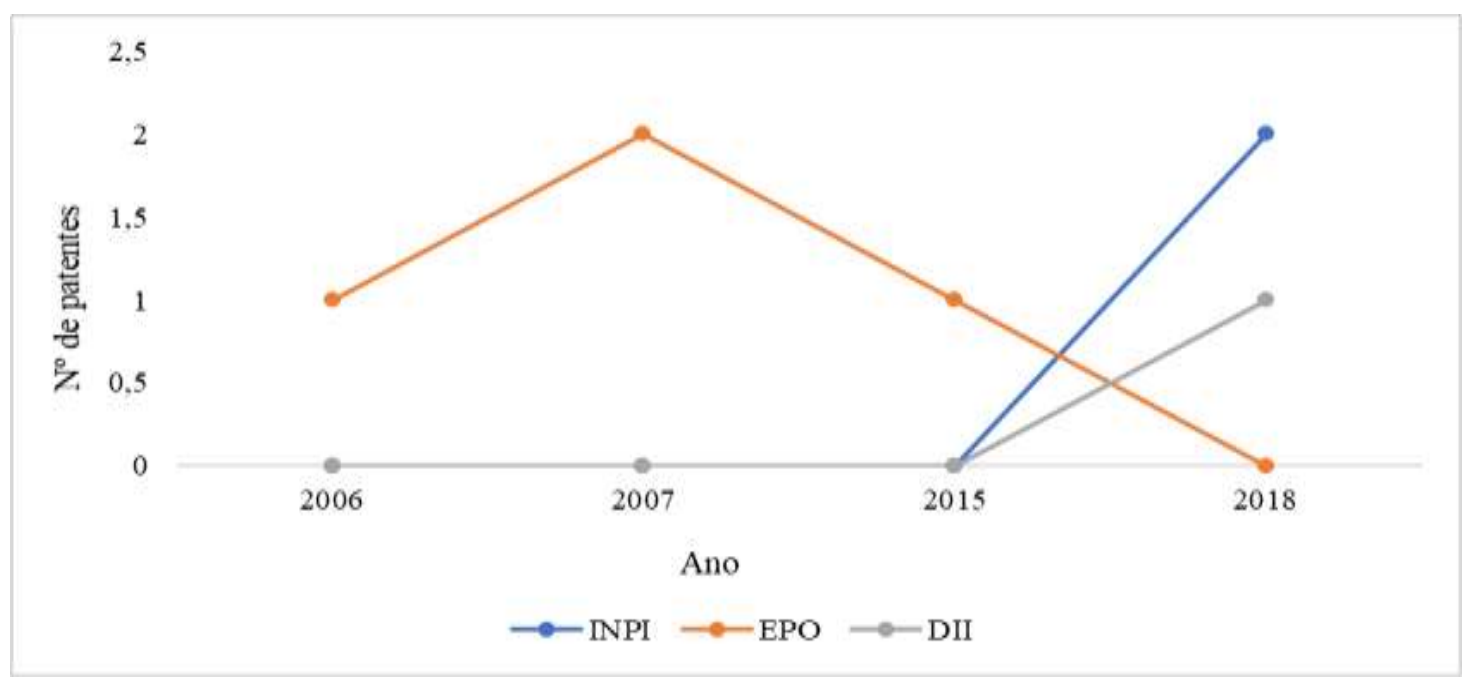

Fonte: Autores (2021). 
Ainda sobre a evolução temporal dos depósitos de patentes, pode-se verificar que não houve registros de 2018 até o presente momento, o que nos permite constatar, mais uma vez, a recorrência de longos intervalos de tempo, uma vez que desde o último registro já se passaram três anos. Por outro lado, esse dado revela a lacuna tecnológica existente para a espécie, evidenciando a necessidade de realização de mais estudos prospectivos ao passo em que demonstra ser um campo de pesquisa promissor, com amplas possibilidades de exploração.

Quanto às instituições depositárias, verificou-se que a maioria das patentes é proveniente de instituições de Ensino Superior brasileiras, com destaque para a Universidade Federal de Campina Grande, com três depósitos, seguida de empresa suíça Ciba Speciality Chemicals Holding INC com dois (Figura 5). Esse dado diverge do cenário internacional, onde segundo Matias-Pereira (2003) e Campos \& Denig (2011), as empresas privadas correspondem aos maiores investidores em pesquisas de desenvolvimento tecnológico, motivados pelo potencial de lucro e competitividade do mercado, enquanto no Brasil são as instituições públicas de ensino, com subsídio governamental, que respondem pelos principais depósitos nas bases de dados.

Figura 5. Perfil dos depositantes.

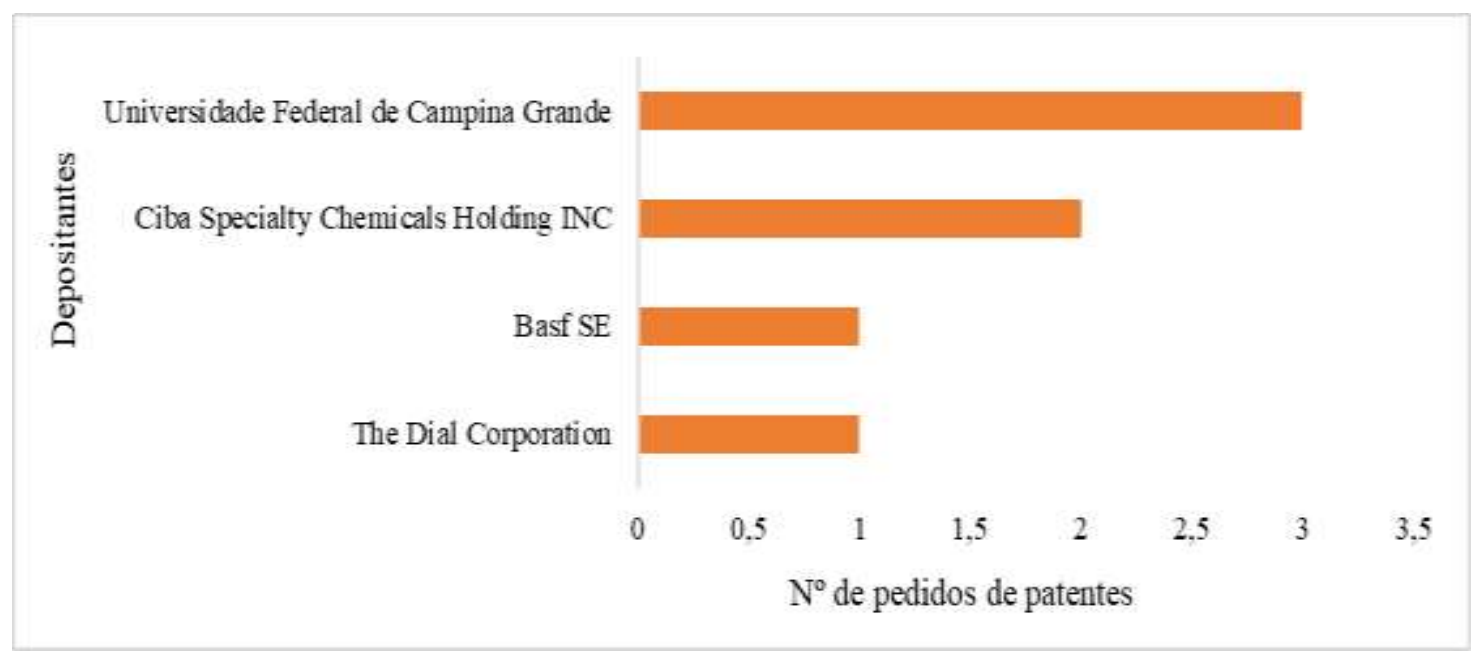

Fonte: Autores (2021).

É importante salientar, que a empresa Ciba atualmente integra o grupo BASF SE, tendo sido incorporada a ele no ano de 2009 (Instituto Observatório Social, 2011). Nesse caso, a multinacional de origem alemã passa a ser detentora do mesmo número de patentes que instituições brasileiras, fazendo com que a liderança tecnológica com uma espécie nativa do Brasil seja compartilhada. Esse resultado demonstra que, mesmo sendo um país com alta diversidade de recursos biológicos, os esforços investidos pelo Brasil não estão sendo capazes de colocá-lo em destaque no mercado internacional, fato que tem dado abertura para que empresas estrangeiras passem a explorar e lucrar com o potencial econômico de espécies brasileiras. No entanto, optou-se por considerar as empresas individualmente, tendo em vista que as patentes analisadas estão registradas de acordo com a configuração empresarial da época em que foram depositadas, dessa forma, o Brasil lidera o ranking.

Em relação à Classificação Internacional de Patentes (CIP), constatou-se que em todas as bases pesquisadas, as patentes pertencem à seção A, que se refere a produtos para necessidades humanas, havendo predomínio das subclasses A23L nas bases INPI e DII e A61K e A61Q na base EPO (Figura 6). A subclasse A23L corresponde alimentos, produtos alimentícios ou bebidas não alcoólicas. A subclasse A61K compreendem preparações para fins médicos, odontológicos ou 
de higiene e A61Q envolve produtos cosméticos e de toalete. Ressalta-se que, dependo da finalidade, a subclasse A61Q pode cobrir tanto usos semelhantes ao do grupo principal A61K e como da subclasse A61P, nesse caso se a preparação for para fins terapêuticos. Independente dessa possível reclassificação, pode-se constatar que, a área de maior desenvolvimento tecnológico para a espécie envolve produtos voltados para fins médicos e cosmetológicos.

Figura 6. Número de patentes por base de dados de acordo com IPC.

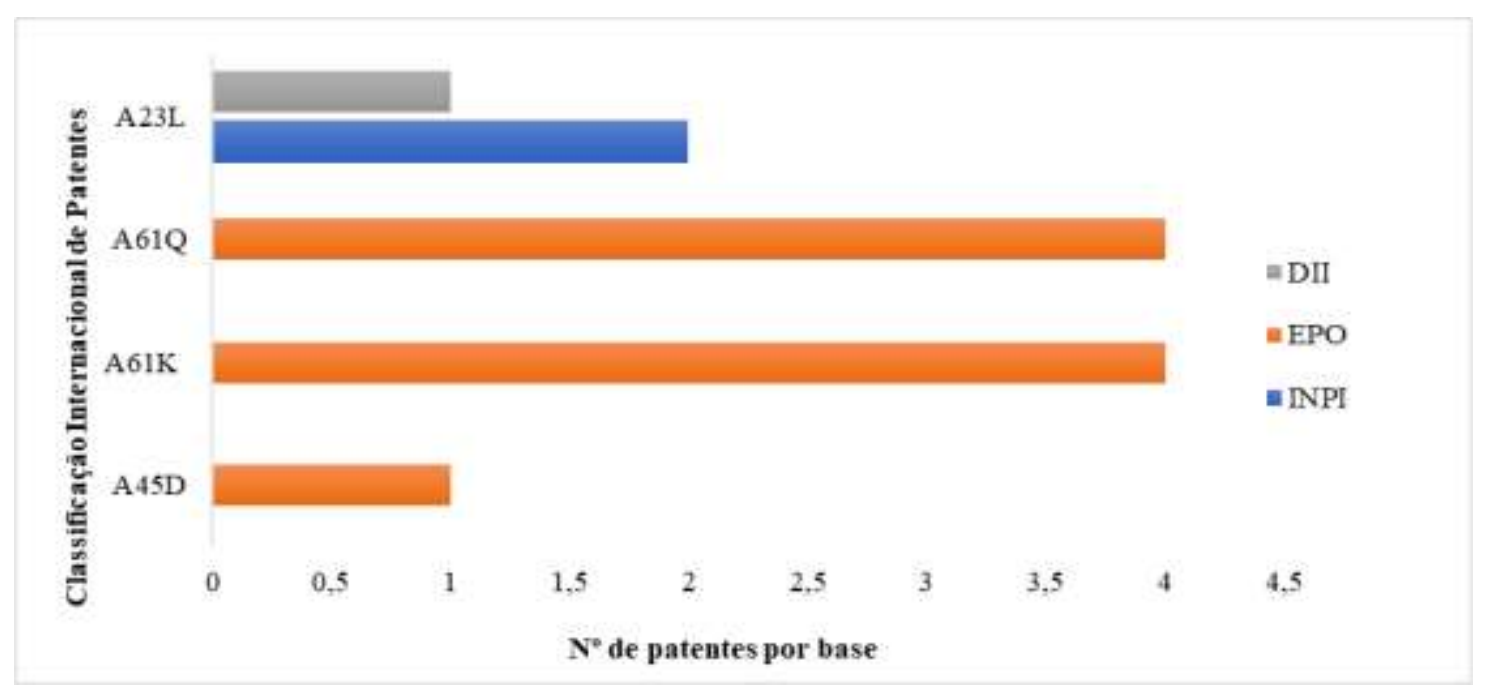

Fonte: Autores (2021).

De acordo com Hiratuka (2008), o mercado de cosméticos, em nível mundial, teve crescimento superior a 5\% sobre o valor no ano de 2007, atingindo US\$290,9 bilhões, em termos de preço de consumidor em 2006. De 2000 para o 2006, o Brasil passou de sexto principal mercado para terceiro, mantendo a posição em 2007, com um crescimento de $22,6 \%$ em relação ao ano anterior. Quanto à representatividade no cenário mundial, o país ocupa a $24^{a}$ posição no ranking dos maiores depositantes, evolução pouco significativa, tendo em vista que essa posição permanece quase inalterada desde 1980, quando o Brasil ocupava a 23ª posição (Griliches, 1990; Bastos \& Jacob, 2017), demonstrando a necessidade de maior incentivo para que possa evoluir no mercado mundial, visto sua posição de destaque em relação à biodiversidade.

\section{Considerações Finais}

A partir deste estudo foi possível determinar o grau de desenvolvimento científico e tecnológico em que se encontra a espécie $C$. coriaceum até o momento, sendo possível constatar que o número de publicações científicas relacionadas à espécie é mais expressivo que o número de patentes depositadas, o que nos permite concluir que, embora ambos os estudos se encontrem em ascensão, mesmo que em ritmo lento, se comparado a outras espécies do gênero, o desenvolvimento científico da espécie encontra-se mais avançado, especialmente em instituições brasileiras. Isso aponta para a necessidade de maior incentivo e investimento em estudos bioprospectivos, a fim de ampliar as tecnologias relacionadas à espécie, fato que pode favorecer o protagonismo do Brasil no mercado internacional, em diversos ramos, visto que a espécie em questão é endêmica do país, onde ainda se encontra significativa parcela da biodiversidade mundial. Além disso, o presente estudo demonstra que as inovações tecnológicas com a espécie estão voltadas basicamente para três finalidades: médicos/odontológicos/higiênicos; cosmetológicas; e alimentícias, revelando que existem outras áreas a serem exploradas.

Para que se tenha um conhecimento mais robusto acerca do C. coriaceum e suas potencialidades, sugere-se que novos estudos sejam realizados, sobretudo nas áreas de concentração não representadas nessa pesquisa, como é o caso da genética e 
ecologia funcional, acerca das quais, não houve registros no presente estudo. Por outro lado, ressalta-se que os dados científicos objetivados e avaliados aqui, se referem a produções de artigos, excetuando-se teses, dissertações e outros tipos de publicações que podem apresentar informações sobre as áreas mencionadas acima. Nesse caso, um estudo prospectivo mais amplo, que contemple os diversos tipos de publicações seria recomendável futura e continuamente, a fim de que se mantenha atualizado, o panorama de publicações acerca da espécie.

\section{Referências}

Amorim, A. M. A., Prance, G. T. \& Medeiros, H. (2012). Caryocaraceae. In: Lista de Espécies da Flora do Brasil, Jardim Botânico do Rio de Janeiro. Jardim Botânico do Rio de Janeiro. http://cncflora.jbrj.gov.br/portal/pt-br/profile/Caryocar\%20coriaceum.

Bastos, V. D. \& Jacob, F. (2017). Resultados Paradoxais da Política de Inovação no Brasil. Revista do BNDES, 47, $359-431$.

Brasil. (2019). Ministério da Agricultura, Pecuária e Abastecimento. Bioeconomia da floresta: a conjuntura da produção florestal não madeireira no Brasil. Serviço Florestal Brasileiro, 45. https://www.florestal.gov.br/documentos/publicacoes/4229-bioeconomia-da-floresta/file.

Brasil. (2006). Ministério da Saúde. Secretaria de Ciência, Tecnologia e Insumos Estratégicos. Política nacional de plantas medicinais e fitoterápicos. Ministério da Saúde, 60. https://bvsms.saude.gov.br/bvs/publicacoes/politica_nacional_fitoterapicos.pdf.

Calixto, J. B. (2003). Biodiversidade como fonte de medicamentos. Ciência e Cultura, 55(3), 37-39.

Campos, A. C. \& Denig, E. A. (2011). Propriedade intelectual: uma análise a partir da evolução das patentes no Brasil. Unioeste, 13(18), 97-120.

CNCFlora. (2012). Caryocar coriaceum. In: Lista Vermelha da flora brasileira versão 2012.2. Centro Nacional de Conservação da Flora. http://cncflora.jbrj.gov.br/portal/pt-br/profile/Caryocar coriaceum.

Galindo-Leal, C. \& Câmara. I. G. (2005). Atlantic forest hotspots status: an overview. In: Galindo-Leal, C., Câmara, I. G. (Ed.). Mata Atlântica: biodiversidade, ameaças e perspectivas (pp. 3-11). São Paulo: Fundação SOS Mata Atlântica.

Griliches, Z. (1990). Patent statistics as economic indicators: a survey. Journal of Economic Literature, 28 (4), $1661-1707$.

Hiratuka, C. (2007). Relatório de Acompanhamento Setorial: Cosméticos. Projeto: Boletim de Conjuntura Industrial, Acompanhamento Setorial e Panorama da Indústria. ABDI/ NEIT/IE/UNICAMP , 1, 1-15.

Instituto Observatório Social. (2011). Responsabilidade social e negociação coletiva na BASF Brasil. 89.

Joly, C. A., Haddad, C. F. B., Verdade, L. M., Oliveira, M. C., Bolzani, V. S. \& Berlinck, R. G. S. (2011). Diagnóstico da pesquisa em biodiversidade no Brasil. Revista USP, (89), 114-133. doi:https://doi.org/10.11606/issn.2316-9036.v0i89p114-133.

Kupfer, D. \& Tigre, P. B. (2004). Prospecção tecnológica. In: Caruso, L. A. \& Tigre, P. B. (Org.). Modelo SENAI de prospecção: documento metodológico. Montevideo, OIT/CINTERFOR, 77.

Lorenzi, H. (2014). Árvores brasileiras: manual de identificação e cultivo de plantas arbóreas nativas do Brasil. Nova Odessa: Plantarum, 384.

Machado, R. B., Ramos-Neto, M.B., Pereira, P. G. P., Caldas, E. F., Gonçalves, D. A., Santos, N. S., Tabor, K. \& Steininger, M. (2004). Estimativas de perda da área do Cerrado brasileiro. Conservação internacional, 1-25.

Matias-Pereira, J. (2003). Política de proteção à propriedade intelectual no Brasil. In: ENANPAD, 27. Anais. Rio de Janeiro, Rio de Janeiro, Brasil. ANPAD, p. 1-15.

Mittermeier, R. A., Turner, W. R., Larsen, F. W., Brooks, T. M. \& Gascon, C. (2011). Global Biodiversity Conservation: The Critical Role of Hotspots. In: Zachos, F., Habel, J. (eds). Biodiversity Hotspots, 3-22.

Myers, N., Mittermeier, R. A., Mittermeier, C. G., Fonseca, G. A. B. \& Kent, J. (2000). Biodiversity hotspots for conservation priorities. Nature, 403, 853858. doi:https://doi.org/10.1038/35002501

Oliveira, D. A., Moreira, P. A., Melo-Júnior, A. F. \& Pimenta, M. A. S. (2006). Potencial da biodiversidade vegetal da Região Norte do Estado de Minas Gerais. Unimontes Científica, 8(1), 23-34.

Oliveira, M. E. B., Guerra, N. B., Barros, L. M. \& Alves, R. E. (2008). Aspectos agronômicos e de qualidade do pequi. Embrapa Agroindústria Tropical, 113, 32 .

Pianovski, A. R., Vilela, A. F. G., Silva, A. A. S., Lima, C. G., Silva, K. K., Carvalho, V. F. M., Musis, C. R., Machado, S. R. P. \& Ferrari, M. (2008). Uso do óleo de pequi (Caryocar brasiliensis) em emulsões cosméticas: desenvolvimento e avaliação da estabilidade física. Revista Brasileira de Ciências Farmacêuticas, 44(2), 249-259.

Pinto, G. (1992). Recursos genéticos de fruteiras nativas na região Nordeste do Brasil. In: Simpósio Nacional de Recursos Genéticos de Fruteiras Nativas. Anais. Cruz das Almas, 81-86.

Prance, G. T. (2007). Ethnobotany, the science of survival: A declaration from Kauai. Economic Botany, 61(1),1-2. 
Research, Society and Development, v. 10, n. 14, e481101422123, 2021

(CC BY 4.0) | ISSN 2525-3409 | DOI: http://dx.doi.org/10.33448/rsd-v10i14.22123

Prance, G.T. \& Pirani, J. R. (2020). Caryocaraceae. http://floradobrasil.jbrj.gov.br/reflora/floradobrasil/FB16719.

In: Flora do Brasil 2020. Jardim Botânico do Rio de Janeiro.

Prance, G. T., \& Marlene Freitas da Silva. (1973). Caryocaraceae. Flora Neotropica, 12, 1-75. http://www.jstor.org/stable/4393688

Ramos, K. M. C. \& Souza, V. A. B. (2011). Características físicas e químico-nutricionais de frutos de pequizeiro (Caryocar coriaceum Wittm.) em populações naturais da região meio-norte do Brasil. Revista Brasileira de Fruticultura, 33(2), 500-508. https://doi.org/10.1590/S0100-29452011005000072

Räsänen, M. E., Linna, A. M., Santos, J. C. R. \& Negri, F. R. (1995). Late Miocene tidal deposits in the Amazonian foreland basin. Science, 269 , 386-389.

Rizzini, C. T. (1978). Árvores e madeiras úteis do Brasil: manual de dendrologia brasileira (2ªed.). São Paulo: Blücher, 312.

Sandes, A. R. R. \& Di Blase, G. (2000). Biodiversidade e diversidade química e genética. In: Biotecnologia, Ciência \& Desenvolvimento 2 (13), 28 - 32.

Scarano, F. R. \& Ceotto, P. (2016). A importância da biodiversidade brasileira e os desafios para a conservação, para a ciência e para o setor privado. In: Rolim, S.G., Menezes, L.F.T. \& Srbek-Araujo, A.C (edi.). Floresta Atlântica de Tabuleiro: diversidade e endemismos na Reserva Natural Vale. Editora Rona Editors, 483-495

Silva, M. A. P. \& Medeiros Filho, S. (2006), Morfologia de fruto, semente e plântula de piqui (Caryocar coriaceum Wittm.). Revista Ciência Agronômica, 37(3), 320-325

Sousa, E. P., Queiroz, A. J. M., Figueirêdo, R. M. F. \& Lemos, D. M. (2014). Comportamento reológico e efeito da temperatura da polpa de pequi em diferentes concentrações. Brazilian. Journal of Food Technology, 17(3), 226-235. http://dx.doi.org/10.1590/1981-6723.1214

Sousa-Júnior, J. R., Collevatti, R. G., Lins-Neto, E. M. F., Peroni, N. \& Albuquerque, U. P. (2018). Traditional management affects the phenotypic diversity of fruits with economic and cultural importance in the Brazilian Savanna. Agroforestry Systems, 92(1),11-21. https://doi.org/10.1007/s10457-016-0005-1

Souza, J. P., Alves, R. E., Brito, E. S., Nogueira, D. H. \& Lima, J. R. (2013). Estabilidade de produtos de pequi (Caryocar coriaceum Wittm) sob congelamento em diferentes tipos de embalagens. Revista Brasileira de Fruticultura, 35 (4), 971-976. https://doi.org/10.1590/S0100-29452013000400007.

Tigre, P. (2006). Gestão da inovação: a economia da tecnologia no Brasil. Rio de janeiro: Elsevier, 282.

Williams, K.J., Ford, A., Rosauer, D. F., Silva, N., Mittermeier, R., Bruce, C., Larsen, F.W. \& Margules, C. (2011). Forests of east Australia: the 35th biodiversity hotspot. In: Zachos, F.E., Habel, J.C. (Eds.), Biodiversity Hotspots. Springer Publishers, $295-310$. 